\title{
Microbial communities for bioprocessing: lessons learned from nature
}

$$
\text { Xuefeng "Nick” Peng }{ }^{1,2} \text {, Sean P. Gilmore }{ }^{1} \text {, Michelle A. O’Malley"1 }
$$

${ }^{1}$ Department of Chemical Engineering, University of California, Santa Barbara, CA, 93106, USA

${ }^{2}$ Department of Earth Science, University of California, Santa Barbara, CA, 93106, USA

*Corresponding author: Michelle A. O’Malley, email: momalley@engineering.ucsb.edu, telephone: (+1) 805-893-4769; fax: (+1) 805-893-4731

\section{Abstract}

Microbial bioprocessing has evolved from the use of undefined natural consortia to the construction of synthetic communities tailored to specific processes and bioproducts. This evolution is enabled by recent advances in biotechnology, including cultivation of non-model microbes, metabolic engineering, bioinformatics, and numerical modeling. Equipped with these powerful tools, engineers have designed co-cultures and consortia with an expanded set of capabilities, mainly via "bottom-up" approaches that tether isolates together in culture. Here, we present a brief review of the opportunities, challenges, and recent developments in consortiabased bioprocessing with a focus on lignocellulosic biomass conversion. With improved understanding of microbial community composition and function, we further present a vision to harness defined consortia down-selected from nature via "top down" approaches.

\section{Keywords}

Microbial consortia; non-model microbes; synthetic biology; fungi; bacteria; bioprocessing

\section{Introduction}

Microbial bioprocessing is a powerful tool that has transformed nearly every aspect of our lives, from the production of food, fuel, solvents, and drugs, to waste water treatment and remediation. From an engineer's standpoint, we typically think of these products as the outcome of one microbial workhorse, where a single strain has been isolated and manipulated in the laboratory to tackle a specific task. However, history reminds us that undefined mixtures of microbes have been used for millennia to advance society. Africans brewed wine as early as 690 $\mathrm{BC}$ [1]; yeasts in the environment have been used to make bread for millennia [2]. Biogas 
generated from mixed microbial metabolism was even used for heating bath water in Assyria in

35 the 10th century BC, and gave rise to modern anaerobic digestion technologies [3]. In the 1970s, oil spills were first remediated by stimulating the growth of naturally occurring microorganisms in the ocean [4]. Rather than building multiple functions into a single microbe, these "top-down" approaches utilize undefined microbial communities that divide-and-conquer difficult metabolic tasks to enable or accelerate outcomes.

\section{Consortia-Enabled Biofuels: An Alternative to CBP}

Conventional methods of biofuel production are usually energy intensive, requiring physical and/or chemical pre-treatment of biomass before enzymatic hydrolysis and microbial fermentation $[9,10]$. Consolidated bioprocessing (CBP) has been proposed as an alternative to conventional methods, and it combines cellulase production, biomass hydrolysis, and sugar fermentation into one step [11]. Classical CBP aims to bypass pretreatment and convert lignocellulosic feedstock into desired products by using one microorganism, such as the yeast Saccharomyces cerevisiae [12-14]. However, it has become clear in the past decade that a multitude of challenges make it difficult to engineer a "superbug" microbe that both breaks down 
and converts biomass into fuels efficiently at industrial scale [15]. Major issues include the difficulty of establishing genetic systems in novel organisms, relatively low specific activity of recombinantly expressed enzymes (including activity against crude substrates), and limited tolerance to concentrated byproducts such as ethanol [15].

A promising alternative to classical CBP is consortia-based bioprocessing, a field of research that has made significant progress recently [16,17]. A microbial consortium consists of more than one strain of microorganism, often with complementary metabolic functions, where difficult tasks can be divided across a diverse subset of microbes. A defined consortium is usually constructed with via a "bottom-up" approach, co-culturing two (or more) isolated strains that have been well characterized (Figure 1). At the other extreme, undefined consortia originate from environmental microbial communities with an unknown number of constituents (Figure 1). However, defined consortia can also be constructed by a "top-down" approach that enriches a stable microbial community, where each member is later isolated and subsequently characterized (Figure 1).

\section{“Bottom-up" Approaches: Advancements and Opportunities}

Most progress in consortia-based bioprocessing to date is based on a "bottom-up" methodology, where synthetic communities of microorganisms have been constructed to accomplish specific goals (Figure 1). Members in these microbial consortia often have a commensal or syntrophic relationship, i.e. the products made by one member are beneficial for and/or used as substrates by another member. A number of synthetic consortia have proven to be more efficient in bioprocessing than mono-cultures [7]. For example, $\mathrm{Xu}$ and Tschirner [18] demonstrated improved efficiency (up to two-fold) of ethanol production by a co-culture of two strains of fermentative Clostridium compared to mono-cultures. They hypothesized that the observed synergy was a result of Clostridium thermolacticum utilizing the degraded substrates from Clostridium thermocellum, which are less favorable for C. thermocellum. Similarly, Zuroff et al. [19] established symbiosis between $C$. phytofermentans and a yeast (either S. cerevisiae or Candida molishiana) stable for 50 days. While $C$. phytofermentans is sensitive to oxygen, both yeasts removed oxygen from the co-culture in return for soluble sugars released by $C$. phytofermentans hydrolysis. The ethanol yield from the co-culture of $C$. phytofermentans and $S$. cerevisiae was more than two-fold higher than either of the mono-cultures. One example of a 
cross-kingdom consortium divided the tasks of hydrolysis and fermentation to the fungus Trichoderma reesei and the bacterium Escherichia coli, respectively [20]. This stable co-culture converted microcrystalline cellulose and pretreated corn stover to isobutanol, yielding $62 \%$ of the theoretical maximum.

While mixed microbial consortia generally outperform a single microorganism, their full potential for bioprocessing has yet to be realized for cost-efficient industrial scale applications. To optimize synthetic microbial consortia, numerical modeling has become an important tool for prediction of metabolic compatibility and consortia design [20-22]. For example, a genomescale metabolic model of $C$. cellulolyticum can successfully predict chemostat growth and byproduct secretion, and it can be used to develop a dynamic model of metabolic interactions in the co-culture with $C$. acetobutylicum [23]. Experimental data have confirmed that modelling frameworks incorporating the interspecies exchange of metabolites predicts the species ratio in microbial consortia [20,24], but these models usually make simplistic assumptions such as maximizing total biomass, which sometimes lead to inaccurate predictions [25]. Zomorrodi [26] showed that it is important to include species- and community- level fitness functions when modeling microbial communities. In addition to genome-scale metabolic modeling, a simpler coarse-grained model, which is tailored to solve the inference problem from the experimental data, has been proposed as a key to understanding microbial communities [27].

In addition to numerical modeling, the incorporation of metabolic engineering promises to offer greater flexibility to control the behavior of synthetic microbial consortia. For example, Shin et al. [28] engineered an E. coli strain to co-express and secrete xylanase and acetylxylan esterase, hydrolyzing xylan in growth media into xylooligosaccharides. A second engineered $E$. coli strain assimilated xylooligosaccharides and converted them into ethanol. This modularly designed co-culture reached $55 \%$ of the theoretical maximum for ethanol yield. Another application of metabolic engineering split the signal production and sensing components originally from Staphylococcus aureus between two strains of Bacillus megaterium [29]. Successfully engineered cell-cell communications in both Gram-positive and Gram-negative hosts [29-31] could be extended to achieve dynamic control of biomass-degrading consortia. Additionally, such a strategy provides an inherent advantage of avoiding the need to monitor cell growth and add exogenous molecules at a specific cell density, and the modular design allows for tuning of each member of the synthetic co-culture [29]. Compared to classical CBP, which 
usually attempts to constitutively overexpress or knock out select genes in one organism, consortia-based metabolic engineering could increase process efficiency and productivity by dividing metabolic burdens between consortia members.

Although synthetic microbial consortia constructed from "bottom-up" indubitably represent a step forward, this approach still faces a number of major challenges. First and foremost is the inability to co-culture microorganisms that are known to form stable and functional communities a priori. More than $99 \%$ of all microorganisms have not been cultivated or genomically characterized, thus the "bottom-up" construction of synthetic consortia is inherently limited by knowledge of characterized strains. Additionally, considering that most model organisms are well domesticated to thrive independently under laboratory conditions, they may not be the best candidates to form robust, stable consortia with other microorganisms. The underlying mechanisms for synergistic effects of synthetic consortia are primarily hypothesized based on the knowledge about their metabolic pathways. In addition to the example of a coculture of T. reesei and E. coli mentioned above [20], such limitations were apparent in the study of a Clostridia co-culture that was constructed to convert filter paper to hydrogen [32]. In this co-culture, $C$. thermopalmarium depends on the hydrolytic products from C. thermocellum, and enhances the overall yield of hydrogen compared to the mono-culture of $C$. thermocellum. However, lack of knowledge regarding the underlying mechanisms of the interactions in this microbial consortium creates obstacles for further engineering efforts such as enhancing stability and scaling up. Moreover, besides the division of labor, it is equally critical to consider the spatial and temporal organization and cumulative behavior as a function of interactions when constructing and evaluating microbial consortia [33].

\section{“Top-down" Approaches: Exploiting Natural Partnerships}

Natural consortia of microorganisms have been applied in multiple contexts, including the conversion of biomass to fuels. Biogas produced from anaerobic digestion is one of the most broadly adopted biofuels in developing countries, with 27 million biogas plants in China, and 4 million in India [34]. Despite the significant economic and environmental benefits offered by biogas plants, there is limited knowledge about the parameters controlling their efficiency and reliability, because they are usually regarded as a "black box" of unknown microbes [35]. 
The first step towards cracking open and understanding these complex systems is to 158 investigate the natural consortia of microorganisms inside. Recent advances in sequencing technology have enabled us to start filling the gaps of our knowledge about the composition and

160 161 diversity of natural microbial communities. In an agricultural biogas plant, 454-pyrosequencing of the total microbial community found that Firmicutes, followed by Bacteroidetes and Proteobacteria were the dominant bacterial members responsible for polysaccharide degradation, while Methanomicrobiales represent the most abundant group of methanogenic archaea [36]. Similar findings were reported in a biogas-producing anaerobic digestion experiment, where the most abundant members of microbial community were the species Methanoculleus marisnigri and from the class Clostridia, as determined by short-read next generation DNA sequencing [37]. Additionally, an extensive temporal pyrosequencing dataset from anaerobic digestion facilities demonstrated the unprecedented level of stability maintained by the resilience of syntrophic bacterial communities [38].

The remarkable stability and resilience observed in these systems stems from a few key factors. First, diversity of a microbial consortium usually translates into functional redundancy. In the event of environmental perturbation, some members of the microbial consortium may experience lowered activity or a reduction in population size. Redundant members may respond positively to make up for the loss of function for the entire consortium [39]. Another stabilizing mechanism is the network of interactions between organisms [39]. Therefore, it is highly attractive to extract a defined microbial consortium from an existing complex consortium. This should retain part of the network of interactions between the members and the corollary stability selected by nature. On the other hand, from an engineering perspective, a microbial consortium with a small number of members should be easier to control than a complex consortium.

Compared to earlier efforts aimed at constructing synthetic microbial consortia from well-studied model organisms, extracting a defined consortium from nature offers a number of advantages. The classic approach to synthesize a consortium for biomass conversion is to select a strain that is capable of breaking down biomass into simple compounds that can be converted by another strain into desired products. However, it is generally impractical to test all possible combinations considering the sheer number of theoretically viable pairs. A better approach is to enrich for naturally occurring microbial consortia from environmental samples, such as the rumen and fecal material of herbivores, under culture conditions that will eventually be used for 
188

189

190

191

192

193

194

195

196

197

198

199

200

201

202

203

204

205

206

207

208

209

210

211

212

213

214

215

216

217

218

production (e.g. [40-42]). The enrichment approach generally ends up with a minimal set of microbes that are highly stable, each of which could be isolated for genomic and transcriptomic analysis, as well as physiological characterization (Figure 1). On the other hand, this "top-down" approach is limited by the lack of established genetic engineering tools for microbes obtained through enrichment. Therefore, the development of a genetic system for these isolated members should set a foundation for later metabolic engineering, enabling another level of control on the performance of and interactions within the consortium.

\section{The Case for Exploiting the Herbivore Gut Microbiota}

One of the best examples of natural consortia that hydrolyze and ferment biomass is found in the herbivore gut. Microorganisms living in the digestive systems of natural grazers have co-evolved with their host for millions of years to utilize lignocellulosic hydrolysates. Microbes span a range of bacteria, fungi, protozoa, and methanogenic archaea [43-45], and the plethora of biomass-degrading genes and genomes discovered from cow rumen indicate that herbivorous grazers' digestive systems should be an ideal source for down-selecting lignocellulolytic consortia [46,47]. Indeed, members from all four types of microorganisms mentioned above have been isolated and characterized from the cow rumen [48-51], and there is a body of literature on mixed cultures of bacteria and archaea (e.g. [52-55]). However, studies exploring the potential of directly down-selected co-cultures including eukaryotic microorganisms remain limited.

The mutualistic paring of fungi and methanogens is one of the consortia achieved by enrichment techniques [41,42]. The primary metabolites of anaerobic fungi include carbon dioxide, hydrogen, and volatile fatty acids, which in turn are utilized by methanogens for growth and methane production. Although anaerobic fungi are outnumbered by their bacterial counterparts in the rumen, there is evidence that fungal populations play a major role in the breakdown of cell walls [56-59]. Flagellated fungal zoospores attach themselves and develop hyphae that penetrate into plant tissues [60]. The physical breakdown of biomass coupled to the array of lignocellulolytic enzymes produced by these fungi [61] ensures efficient degradation of biomass. For example, anaerobic fungal enzymes are rich in xylan-degrading enzymes and hence unbiased in substrate preference [61]. Therefore, a wide variety of feedstocks that are already produced and often considered as "waste" such as corn stover can be used for methane 
production. This saves arable land for cultivation of food rather than dedicating them for biofuel production as in the case of corn to ethanol, which is one of the two largest bio-ethanol programs in the world [62]. The other major bio-ethanol program extracts juice from sugar cane as a sugar source for fermentation, and the bagasse is either burnt for additional energy or simply discarded [63]. It has been estimated that if 50\% of the bagasse after juice extraction is converted to ethanol, potentially using a microbial consortium, ethanol yield per hectare of land can increase from 6,000 L/ha to $10,000 \mathrm{~L} /$ ha [64]. Moreover, the efficient conversion of lignocellulosic biomass to biofuels should reduce the burden of disposing the agricultural "waste" products and lessens their environmental impact, serving as a solution to the "food, energy, and environment trilemma" [62].

\section{Conclusions and Perspectives}

Humanity has, knowingly or unknowingly, harnessed the power of microbes and microbial communities for bioprocessing since ancient times. More recently, we have come to recognize that microbial processing ranges between two extremes: from microbial communities that are "black boxes" to single microbe mediated fermentations. While the use of "top-down" approaches exploit nature to accomplish difficult tasks, control of these communities is limited due to lack of understanding of the microbial community composition, metabolism, and dynamics. The advance of new tools, including metabolic engineering of non-model hosts, sequencing and bioinformatics, and numerical modeling, have enabled engineers to parse the roles of community members like never before to understand their function. With these tools in hand, synthetic co-cultures can be constructed from the "bottom-up" to capture the behavior of natural microbial communities. Considering the strengths and weakness of both "top-down" and "bottom-up" approaches, the "top-down" approach is likely advantageous when the goal focuses on the output and stability of microbial processing and the ability to control each member of the microbial consortia is less of a concern. On the other hand, the "bottom-up" approach is likely favorable when the ability to manipulate each member of the consortia is necessary.

Along these lines, defined consortia down-selected from environmental samples are a highly attractive compromise to synthetic co-cultures - combining the advantages of both "topdown" and "bottom-up" approaches. However, this methodology still faces a number of major challenges, including the resistance to cultivation by many microorganisms, the difficulty in 
developing novel genetic systems for metabolic engineering, and deciphering the genetic codes and interwoven metabolism of the microbial consortia. To move the field forward, it is critical to integrate all tools at hand to make informed decisions, with a focus on selecting stable consortia that have the ability to thrive at industrial scale. Genomic and transcriptomic characterization of each member of a defined consortium allows for the discovery of metabolic pathways and potentially their controls, which lays the foundation for system optimization, especially when combined with numerical modeling. Insights gained through metagenomic and metatranscriptomic analysis of natural microbial communities should further guide the enrichment strategy when down-selecting microbial consortia, particularly for applications relevant to biomass hydrolysis and product fermentation.

\section{Acknowledgements}

The authors acknowledge support from the California NanoSystems Institute (CNSI), supported by the University of California, Santa Barbara and the University of California, Office of the President. This work was also supported by the Office of Science (BER), U.S. Department of Energy (DE-SC0010352), the Institute for Collaborative Biotechnologies through grant W911NF-09-0001, and the National Science Foundation (MCB-1553721). SPG is a recipient of the National Science Foundation Graduate Research Fellowship Program under Grant No. DGE 1144085.

\section{References}

\section{(*) Papers of special interest}

Solomon et al. 2016

By analyzing the transcriptomes of previously uncharacterized anaerobic gut fungi, this study discovered a large, comprehensive array of unbiased, biomass-degrading enzymes that synergistically degrade crude, untreated plant biomass, and are competitive with optimized commercial preparations from Aspergillus and Trichoderma.

Solomon et al. 2014

This paper summarizes the practice of using next-generation sequencing, proteomics, and bioinformatics to derive biological insight from complex microbial communities, including their composition and function.

\section{Haitjema et al. 2014}


This paper details recent methodological progress in the study of anaerobic gut fungi. Specifically, advances in isolation, culture, and cellulolytic enzyme discovery should promote bioengineering efforts to adapt these non-model organisms for biofuel production.

\section{Marchand and Collins 2015}

This study was the first to engineer synthetic quorum sensing and cell-cell communication system in a Gram-positive host, Bacillus megaterium. It also split the signal production and sensing components between two strains of $B$. megaterium. It has the potential to enable the generation of dynamic gene regulatory networks in B. megaterium and other Grampositive strains.

\section{Hoffner and Barton 2014}

This study outlines a roadmap towards the quantitative design and optimization of low cost resilient artificial ecologies based on microbial consortia, using algal production of fuels and chemicals as an example. The proposed numerical model integrates metabolic information with the ecological scale of the inter-species interactions and the process scale of bioreactors.

\section{Konopka et al. 2015}

This paper identified principles behind the functional stability and robustness in microbial communities. The authors pointed out that the network of interactions between organisms provides a buffer against disturbance beyond the effect of functional redundancy, as alternative pathways with different combinations of microbes can be recruited to fulfill specific functions.

[1] S.A. Odunfa, O.B. Oyewole, African fermented foods, in: B.J.B. Wood (Ed.), Microbiol. Fermented Foods, Springer US, 1998: pp. 713-752. http://link.springer.com/chapter/10.1007/978-1-46130309-1_23 (accessed May 13, 2016).

[2] E. Caplice, G.F. Fitzgerald, Food fermentations: role of microorganisms in food production and preservation, Int. J. Food Microbiol. 50 (1999) 131-149. doi:10.1016/S0168-1605(99)00082-3.

[3] P.J. He, Anaerobic digestion: An intriguing long history in China, Waste Manag. 30 (2010) 549-550. doi:10.1016/j.wasman.2010.01.002.

[4] R.M. Atlas, R. Bartha, Degradation and mineralization of petroleum in sea water: Limitation by nitrogen and phosphorous, Biotechnol. Bioeng. 14 (1972) 309-318. doi:10.1002/bit.260140304.

[5] C. Walsh, Antibiotics, American Society of Microbiology, 2003. http://www.asmscience.org/content/book/10.1128/9781555817886 (accessed May 13, 2016).

[6] U. Van Dongen, M.S.M. Jetten, M.C.M. Van Loosdrecht, The SHARON ${ }^{\circledR}$-Anammox ${ }^{\circledR}$ process for treatment of ammonium rich wastewater, in: Water Sci. Technol., International Water Association, 2001: pp. 153-160. http://cat.inist.fr/?aModele=afficheN\&cpsidt=1019042 (accessed May 13, 2016).

[7] T.R. Zuroff, W.R. Curtis, Developing symbiotic consortia for lignocellulosic biofuel production, Appl. Microbiol. Biotechnol. 93 (2012) 1423-1435. doi:10.1007/s00253-011-3762-9.

[8] A. Demirbas, Political, economic and environmental impacts of biofuels: A review, Appl. Energy. 86, Supplement 1 (2009) S108-S117. doi:10.1016/j.apenergy.2009.04.036. 
[9] A. McAloon, F. Taylor, W. Yee, K. Ibsen, R. Wooley, Determining the Cost of Producing Ethanol from Corn Starch and Lignocellulosic Feedstocks, National Renewable Energy Lab., Golden, CO (US), 2000. http://www.osti.gov/scitech/biblio/766198 (accessed May 16, 2016).

[10] N. Mosier, C. Wyman, B. Dale, R. Elander, Y.Y. Lee, M. Holtzapple, M. Ladisch, Features of promising technologies for pretreatment of lignocellulosic biomass, Bioresour. Technol. 96 (2005) 673-686. doi:10.1016/j.biortech.2004.06.025.

[11] L.R. Lynd, P.J. Weimer, W.H. van Zyl, I.S. Pretorius, Microbial Cellulose Utilization: Fundamentals and Biotechnology, Microbiol. Mol. Biol. Rev. 66 (2002) 506-577. doi:10.1128/MMBR.66.3.506577.2002.

[12] R. Den Haan, J.E. Mcbride, D.C.L. Grange, L.R. Lynd, W.H. Van Zyl, Functional expression of cellobiohydrolases in Saccharomyces cerevisiae towards one-step conversion of cellulose to ethanol, Enzyme Microb. Technol. 40 (2007) 1291-1299. doi:10.1016/j.enzmictec.2006.09.022.

[13] W.H. van Zyl, L.R. Lynd, R. den Haan, J.E. McBride, Consolidated Bioprocessing for Bioethanol Production Using Saccharomyces cerevisiae, in: L. Olsson (Ed.), Biofuels, Springer Berlin Heidelberg, 2007: pp. 205-235. http://link.springer.com/chapter/10.1007/10_2007_061 (accessed June 8, 2016).

[14] R. Den Haan, S.H. Rose, L.R. Lynd, W.H. van Zyl, Hydrolysis and fermentation of amorphous cellulose by recombinant Saccharomyces cerevisiae, Metab. Eng. 9 (2007) 87-94. doi:10.1016/j.ymben.2006.08.005.

[15] D.G. Olson, J.E. McBride, A. Joe Shaw, L.R. Lynd, Recent progress in consolidated bioprocessing, Curr. Opin. Biotechnol. 23 (2012) 396-405. doi:10.1016/j.copbio.2011.11.026.

[16] K. Brenner, L. You, F.H. Arnold, Engineering microbial consortia: a new frontier in synthetic biology, Trends Biotechnol. 26 (2008) 483-489. doi:10.1016/j.tibtech.2008.05.004.

[17] J. Shong, M.R. Jimenez Diaz, C.H. Collins, Towards synthetic microbial consortia for bioprocessing, Curr. Opin. Biotechnol. 23 (2012) 798-802. doi:10.1016/j.copbio.2012.02.001.

[18] L. Xu, U. Tschirner, Improved ethanol production from various carbohydrates through anaerobic thermophilic co-culture, Bioresour. Technol. 102 (2011) 10065-10071. doi:10.1016/j.biortech.2011.08.067.

[19] T.R. Zuroff, S.B. Xiques, W.R. Curtis, Consortia-mediated bioprocessing of cellulose to ethanol with a symbiotic Clostridium phytofermentans/yeast co-culture, Biotechnol. Biofuels. 6 (2013) 59. doi:10.1186/1754-6834-6-59.

[20] J.J. Minty, M.E. Singer, S.A. Scholz, C.-H. Bae, J.-H. Ahn, C.E. Foster, J.C. Liao, X.N. Lin, Design and characterization of synthetic fungal-bacterial consortia for direct production of isobutanol from cellulosic biomass, Proc. Natl. Acad. Sci. 110 (2013) 14592-14597. doi:10.1073/pnas.1218447110.

[21] K. Höffner, P.I. Barton, Design of Microbial Consortia for Industrial Biotechnology, in: J.D.S. and G.P.T. Mario R. Eden (Ed.), Comput. Aided Chem. Eng., Elsevier, 2014: pp. 65-74. http://www.sciencedirect.com/science/article/pii/B9780444634337500080 (accessed May 13, 2016).

[22] T.J. Hanly, M.A. Henson, Unstructured Modeling of a Synthetic Microbial Consortium for Consolidated Production of Ethanol, IFAC Proc. Vol. 46 (2013) 157-162. doi:10.3182/20131216-3IN-2044.00003.

[23] F. Salimi, K. Zhuang, R. Mahadevan, Genome-scale metabolic modeling of a clostridial co-culture for consolidated bioprocessing, Biotechnol. J. 5 (2010) 726-738. doi:10.1002/biot.201000159.

[24] W.R. Harcombe, W.J. Riehl, I. Dukovski, B.R. Granger, A. Betts, A.H. Lang, G. Bonilla, A. Kar, N. Leiby, P. Mehta, C.J. Marx, D. Segrè, Metabolic Resource Allocation in Individual Microbes Determines Ecosystem Interactions and Spatial Dynamics, Cell Rep. 7 (2014) 1104-1115. doi:10.1016/j.celrep.2014.03.070. 
[25] T.J. Hanly, M.A. Henson, Dynamic model-based analysis of furfural and HMF detoxification by pure and mixed batch cultures of S. cerevisiae and S. stipitis, Biotechnol. Bioeng. 111 (2014) 272-284. doi:10.1002/bit.25101.

[26] A.R. Zomorrodi, M.M. Islam, C.D. Maranas, d-OptCom: Dynamic Multi-level and Multi-objective Metabolic Modeling of Microbial Communities, ACS Synth. Biol. 3 (2014) 247-257. doi:10.1021/sb4001307.

[27] M. Hanemaaijer, W.F.M. Röling, B.G. Olivier, R.A. Khandelwal, B. Teusink, F.J. Bruggeman, Systems modeling approaches for microbial community studies: from metagenomics to inference of the community structure, Syst. Microbiol. 6 (2015) 213. doi:10.3389/fmicb.2015.00213.

[28] H.-D. Shin, S. McClendon, T. Vo, R.R. Chen, Escherichia coli Binary Culture Engineered for Direct Fermentation of Hemicellulose to a Biofuel, Appl. Environ. Microbiol. 76 (2010) 8150-8159. doi:10.1128/AEM.00908-10.

[29] N. Marchand, C.H. Collins, Synthetic Quorum Sensing and Cell-Cell Communication in GramPositive Bacillus megaterium, ACS Synth. Biol. (2015). doi:10.1021/acssynbio.5b00099.

[30] H. Kobayashi, M. Kærn, M. Araki, K. Chung, T.S. Gardner, C.R. Cantor, J.J. Collins, Programmable cells: Interfacing natural and engineered gene networks, Proc. Natl. Acad. Sci. U. S. A. 101 (2004) 8414-8419. doi:10.1073/pnas.0402940101.

[31] N. Marchand, C.H. Collins, Peptide-based communication system enables Escherichia coli to Bacillus megaterium interspecies signaling, Biotechnol. Bioeng. 110 (2013) 3003-3012. doi:10.1002/bit.24975.

[32] A. Geng, Y. He, C. Qian, X. Yan, Z. Zhou, Effect of key factors on hydrogen production from cellulose in a co-culture of Clostridium thermocellum and Clostridium thermopalmarium, Bioresour. Technol. 101 (2010) 4029-4033. doi:10.1016/j.biortech.2010.01.042.

[33] S.R. Lindemann, H.C. Bernstein, H.-S. Song, J.K. Fredrickson, M.W. Fields, W. Shou, D.R. Johnson, A.S. Beliaev, Engineering microbial consortia for controllable outputs, ISME J. (2016). doi:10.1038/ismej.2016.26.

[34] T. Bond, M.R. Templeton, History and future of domestic biogas plants in the developing world, Energy Sustain. Dev. 15 (2011) 347-354. doi:10.1016/j.esd.2011.09.003.

[35] P. Weiland, Biogas production: current state and perspectives, Appl. Microbiol. Biotechnol. 85 (2009) 849-860. doi:10.1007/s00253-009-2246-7.

[36] S. Jaenicke, C. Ander, T. Bekel, R. Bisdorf, M. Dröge, K.-H. Gartemann, S. Jünemann, O. Kaiser, L. Krause, F. Tille, M. Zakrzewski, A. Pühler, A. Schlüter, A. Goesmann, Comparative and Joint Analysis of Two Metagenomic Datasets from a Biogas Fermenter Obtained by 454-Pyrosequencing, PLOS ONE. 6 (2011) e14519. doi:10.1371/journal.pone.0014519.

[37] R. Wirth, E. Kovács, G. Maróti, Z. Bagi, G. Rákhely, K.L. Kovács, Characterization of a biogasproducing microbial community by short-read next generation DNA sequencing, Biotechnol. Biofuels. 5 (2012) 41. doi:10.1186/1754-6834-5-41.

[38] J.J. Werner, D. Knights, M.L. Garcia, N.B. Scalfone, S. Smith, K. Yarasheski, T.A. Cummings, A.R. Beers, R. Knight, L.T. Angenent, Bacterial community structures are unique and resilient in fullscale bioenergy systems, Proc. Natl. Acad. Sci. 108 (2011) 4158-4163. doi:10.1073/pnas.1015676108.

[39] A. Konopka, S. Lindemann, J. Fredrickson, Dynamics in microbial communities: unraveling mechanisms to identify principles, ISME J. 9 (2015) 1488-1495. doi:10.1038/ismej.2014.251.

[40] K.N. Joblin, H. Matsui, G.E. Naylor, K. Ushida, Degradation of fresh ryegrass by methanogenic cocultures of ruminal fungi grown in the presence or absence of Fibrobacter succinogenes, Curr. Microbiol. 45 (2002) 46-53. doi:10.1007/s00284-001-0078-5.

[41] Y.F. Cheng, J.E. Edwards, G.G. Allison, W.-Y. Zhu, M.K. Theodorou, Diversity and activity of enriched ruminal cultures of anaerobic fungi and methanogens grown together on lignocellulose in 
461

462

463

464

465

466

467

468

consecutive batch culture, Bioresour. Technol. 100 (2009) 4821-4828. doi:10.1016/j.biortech.2009.04.031.

[42] W. Jin, Y.-F. Cheng, S.-Y. Mao, W.-Y. Zhu, Isolation of natural cultures of anaerobic fungi and indigenously associated methanogens from herbivores and their bioconversion of lignocellulosic materials to methane, Bioresour. Technol. 102 (2011) 7925-7931. doi:10.1016/j.biortech.2011.06.026.

[43] R.E. Hungate, The rumen and its microbes, Academic Press, 1966.

[44] T. Bauchop, R.T. Clarke, Attachment of the ciliate Epidinium Crawley to plant fragments in the sheep rumen., Appl. Environ. Microbiol. 32 (1976) 417-422.

[45] D.E. Akin, R. Benner, Degradation of polysaccharides and lignin by ruminal bacteria and fungi., Appl. Environ. Microbiol. 54 (1988) 1117-1125.

[46] M. Hess, A. Sczyrba, R. Egan, T.-W. Kim, H. Chokhawala, G. Schroth, S. Luo, D.S. Clark, F. Chen, T. Zhang, R.I. Mackie, L.A. Pennacchio, S.G. Tringe, A. Visel, T. Woyke, Z. Wang, E.M. Rubin, Metagenomic Discovery of Biomass-Degrading Genes and Genomes from Cow Rumen, Science. 331 (2011) 463-467. doi:10.1126/science.1200387.

[47] M. Morrison, P.B. Pope, S.E. Denman, C.S. McSweeney, Plant biomass degradation by gut microbiomes: more of the same or something new?, Curr. Opin. Biotechnol. 20 (2009) 358-363. doi:10.1016/j.copbio.2009.05.004.

[48] M. Bryant, L. Burkey, Cultural Methods and Some Characteristics of Some of the More Numerous Groups of Bacteria in the Bovine Rumen, J. Dairy Sci. 36 (1953) 205-217.

[49] P. Smith, R. Hungate, Isolation and Characterization of Methanobacterium-Ruminantium N-Sp, J. Bacteriol. 75 (1958) 713-718.

[50] A.G. WILLIAMS, C.G. HARFOOT, Factors Affecting the Uptake and Metabolism of Soluble Carbohydrates by the Rumen Ciliate Dasytricha ruminantium Isolated from Ovine Rumen Contents by Filtration, Microbiology. 96 (1976) 125-136. doi:10.1099/00221287-96-1-125.

[51] K.N. Joblin, Isolation, Enumeration, and Maintenance of Rumen Anaerobic Fungi in Roll Tubes, Appl. Environ. Microbiol. 42 (1981) 1119-1122.

[52] J.G. Ferry, R.S. Wolfe, Anaerobic degradation of benzoate to methane by a microbial consortium, Arch. Microbiol. 107 (1976) 33-40. doi:10.1007/BF00427864.

[53] M. Chen, M.J. Wolin, Influence of $\mathrm{CH} 4$ production by Methanobacterium ruminantium on the fermentation of glucose and lactate by Selenomonas ruminantium., Appl. Environ. Microbiol. 34 (1977) 756-759.

[54] M.J. Latham, M.J. Wolin, Fermentation of cellulose by Ruminococcus flavefaciens in the presence and absence of Methanobacterium ruminantium., Appl. Environ. Microbiol. 34 (1977) 297-301.

[55] P.J. Weimer, J.G. Zeikus, Fermentation of cellulose and cellobiose by Clostridium thermocellum in the absence of Methanobacterium thermoautotrophicum., Appl. Environ. Microbiol. 33 (1977) 289-297.

[56] S.S. Lee, J.K. Ha, K.-J. Cheng, Relative Contributions of Bacteria, Protozoa, and Fungi to In Vitro Degradation of Orchard Grass Cell Walls and Their Interactions, Appl. Environ. Microbiol. 66 (2000) 3807-3813. doi:10.1128/AEM.66.9.3807-3813.2000.

[57] K.V. Solomon, C.H. Haitjema, D.A. Thompson, M.A. O'Malley, Extracting data from the muck: deriving biological insight from complex microbial communities and non-model organisms with next generation sequencing, Curr. Opin. Biotechnol. 28 (2014) 103-110. doi:10.1016/j.copbio.2014.01.007.

[58] C.H. Haitjema, K.V. Solomon, J.K. Henske, M.K. Theodorou, M.A. O'Malley, Anaerobic gut fungi: Advances in isolation, culture, and cellulolytic enzyme discovery for biofuel production, Biotechnol. Bioeng. 111 (2014) 1471-1482. doi:10.1002/bit.25264. 
[59] K.V. Solomon, J.K. Henske, M.K. Theodorou, M.A. O'Malley, Robust and effective methodologies for cryopreservation and DNA extraction from anaerobic gut fungi, Anaerobe. 38 (2016) 39-46. doi:10.1016/j.anaerobe.2015.11.008.

[60] T. Bauchop, Increasing the Nutritive Value of Poor Quality Materials; Chemical, Nutritional and Feeding Aspects of Lignocellulosic WastesThe anaerobic fungi in rumen fibre digestion, Agric. Environ. 6 (1981) 339-348. doi:10.1016/0304-1131(81)90021-7.

[61] K.V. Solomon, C.H. Haitjema, J.K. Henske, S.P. Gilmore, D. Borges-Rivera, A. Lipzen, H.M. Brewer, S.O. Purvine, A.T. Wright, M.K. Theodorou, I.V. Grigoriev, A. Regev, D.A. Thompson, M.A. O'Malley, Early-branching gut fungi possess a large, comprehensive array of biomass-degrading enzymes, Science. (2016) aad1431. doi:10.1126/science.aad1431.

[62] D. Tilman, R. Socolow, J.A. Foley, J. Hill, E. Larson, L. Lynd, S. Pacala, J. Reilly, T. Searchinger, C. Somerville, R. Williams, Beneficial Biofuels-The Food, Energy, and Environment Trilemma, Science. 325 (2009) 270-271. doi:10.1126/science.1177970.

[63] A.E. Wheals, L.C. Basso, D.M.G. Alves, H.V. Amorim, Fuel ethanol after 25 years, Trends Biotechnol. 17 (1999) 482-487. doi:10.1016/S0167-7799(99)01384-0.

[64] C.R. Soccol, L.P. de S. Vandenberghe, A.B.P. Medeiros, S.G. Karp, M. Buckeridge, L.P. Ramos, A.P. Pitarelo, V. Ferreira-Leitão, L.M.F. Gottschalk, M.A. Ferrara, E.P. da Silva Bon, L.M.P. de Moraes, J. de A. Araújo, F.A.G. Torres, Bioethanol from lignocelluloses: Status and perspectives in Brazil, Bioresour. Technol. 101 (2010) 4820-4825. doi:10.1016/j.biortech.2009.11.067. 


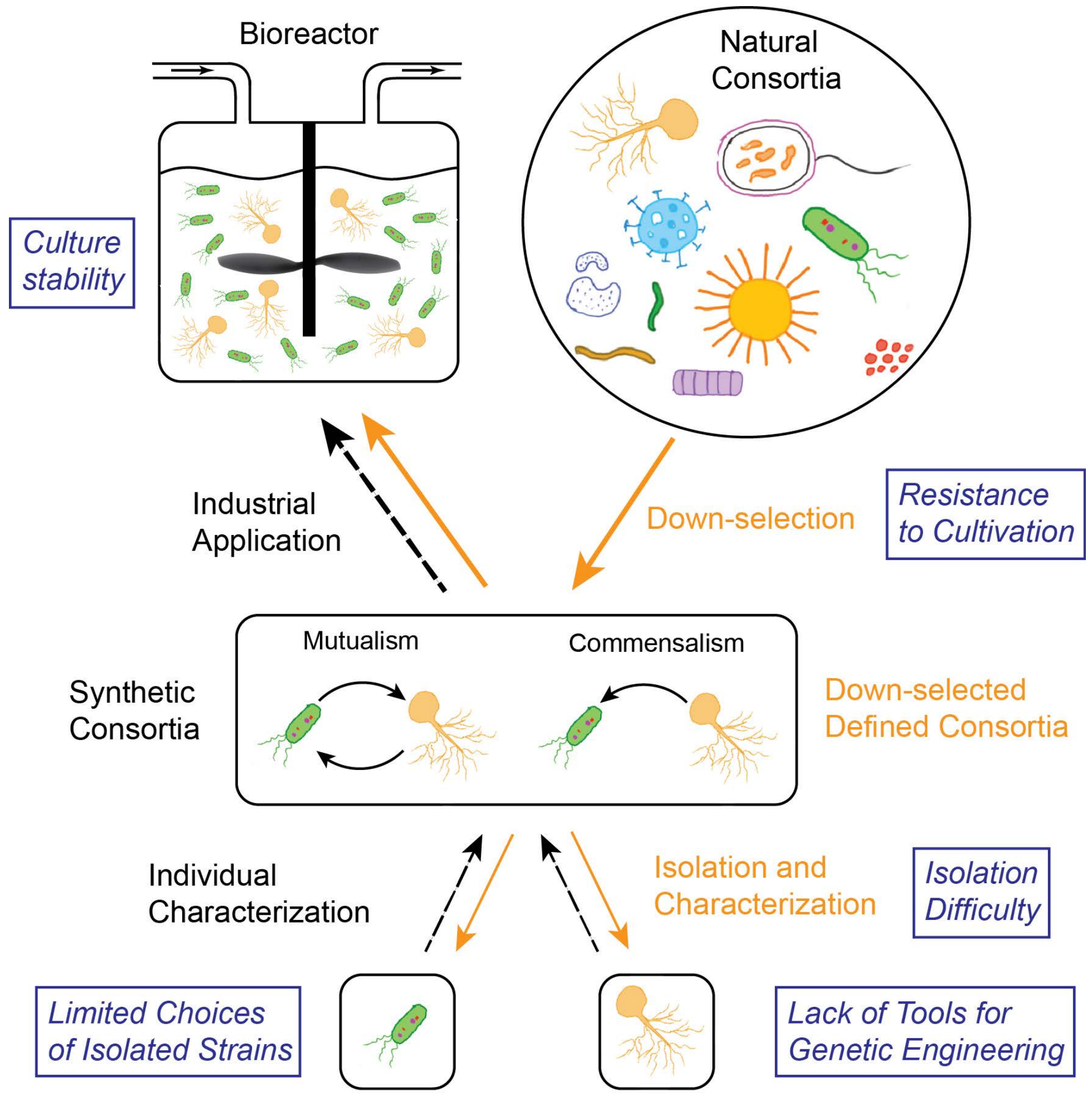

Optional: Metabolic Modeling and Engineering 